\title{
RESEARCH
}

Open Access

\section{Psychometric properties of a condition- specific PROM for the psychosocial consequences of Labelling hypertension by using Rasch analysis}

János Valery Gyuricza ${ }^{1,2^{*}}$ B, Karl Bang Christensen ${ }^{3}$, Ana Flávia Pires Lucas d'Oliveira ${ }^{1}$ and John Brodersen ${ }^{2,4}$

\begin{abstract}
Background: A previous qualitative assessment of the psychosocial consequences of labelling hypertension describes the diagnosis of hypertension as a labelling event with potential unintended negative long-term psychosocial consequences (labelling effects). Until now, the benefits of diagnosing hypertension have been far more reported than the harms. To obtain the net result of the preventive interventions for cardiovascular disease, such as diagnosing and treating mild hypertension, assessing benefits and harms in the most comprehensive way possible is necessary, including the psychosocial consequences of labelling. When measuring psychosocial consequences of labelling hypertension, a questionnaire with high content validity and adequate psychometric properties is needed.
\end{abstract}

Objectives: The aim of this study was to describe the psychometric parameters of face and content-validated pool of items. Other objectives were also to screen the item pool by using Rasch model analysis and confirmatory factor analysis (CFA) for identifying such items with sufficient fit to the hypothesised models.

Methods: We surveyed the pool of items as a draft questionnaire to Brazilians recruited via social networks, sending e-mails, WhatsApp ${ }^{\oplus}$ messages and posting on Facebook ${ }^{\oplus}$. The inclusion criteria were to be older than 18 years old, to be healthy and to have only hypertension.

We used Rasch model analysis to screen the item pool, discarding items that did not fit the hypothesised domain. We searched for local dependence and differential item functioning. We used CFA to confirm the derived measurement models and complementarily assessed reliability using Cronbach's coefficient alpha.

(Continued on next page)

\footnotetext{
* Correspondence: jvgyuricza@usp.br

'Departamento de Medicina Preventiva, Faculdade de Medicina da

Universidade de São Paulo, Av. Dr Arnaldo, 4552 andar. CEP, São Paulo, SP 01246-903, Brazil

${ }^{2}$ Department of Public Health, Section of General Practice and Research Unit for General Practice, University of Copenhagen, Øster Farimagsgade 5,

building 24, P.O. Box 2099, 1014 Copenhagen K, Denmark

Full list of author information is available at the end of the article
}

\section{Springer Open}

( ) The Author(s). 2021 Open Access This article is licensed under a Creative Commons Attribution 4.0 International License, which permits use, sharing, adaptation, distribution and reproduction in any medium or format, as long as you give appropriate credit to the original author(s) and the source, provide a link to the Creative Commons licence, and indicate if changes were made. The images or other third party material in this article are included in the article's Creative Commons licence, unless indicated otherwise in a credit line to the material. If material is not included in the article's Creative Commons licence and your intended use is not permitted by statutory regulation or exceeds the permitted use, you will need to obtain permission directly from the copyright holder. To view a copy of this licence, visit http://creativecommons.org/licenses/by/4.0/. 
(Continued from previous page)

Results: The validation sample consisted of 798 respondents. All 798 respondents completed Part I, whereas 285 (35.7\%) — those with hypertension — completed Part II. A condition-specific questionnaire with high content validity and adequate psychometric properties was developed for people labelled with hypertension. This measure is called 'Consequences of Labelling Hypertension Questionnaire' and covers the psychosocial consequences of labelling hypertension in two parts, encompassing a total of 71 items in 15 subscales and 11 single items.

Conclusion: We developed a tool that can be used in future research involving hypertension, especially in scenarios of screening, prevention, population strategies and in intervention studies. Future use and testing of the questionnaire may still be required.

Keywords: Hypertension, Psychosocial consequences, Patient-reported outcome, Psychometric properties

\section{Background}

Approximately one-fourth of the world's population has blood pressure above the diagnostic threshold for hypertension [1]. Among them, the lowest risk group is that with mild hypertension, which accounts for roughly $60 \%$ of the people diagnosed with hypertension Preventive population strategies may reduce cardiovascular disease (CVD) burden [2]. However, previous studies failed to prove the benefits of the primary prevention of CVD on the basis of a risk strategy-pharmacologic treatmentfor people with mild hypertension [3].

To obtain the net result of preventive interventions for CVD, such as diagnosing and treating mild hypertension, assessing the benefits and harms in the most comprehensive way possible is wise. Until now, studies seem to overlook all possible harms; specifically, psychosocial harms have been far less studied than potential benefits [4].

One unintended harm that has been recognised but has not been comprehensively studied are the negative psychosocial consequences labelling hypertension. Sir George Pickering suggested that hypertension labelling may evoke a feeling of fear of the affliction of a serious disease in a patient [5]. In the next decades, this issue has been addressed in a few studies [6]. One seminal study among Canadian steelworkers $[7,8]$ suggests that after the diagnosis of hypertension, a few negative psychosocial consequences are observed: people experience additional symptoms, increase in absenteeism, become dependent on the healthcare system, worsen their marital relations and are psychologically distressed. The same effects are not observed in those that are unaware of their diagnosis. This study provided relevant insights into the extension of the possible negative effects of labelling but failed to obtain patient-reported outcomes and failed to fully uncover the psychosocial consequences of labelling hypertension. Patient-reported outcomes are reports that come directly from patients about the status of their health condition without the amendment of interpretation of their response by an interviewer [9] and are considered prerequisites for the assessment of psychosocial consequences [10].
A previous qualitative assessment of the psychosocial consequences of labelling hypertension described the diagnosis of hypertension as a labelling event with potential unintended negative long-term psychosocial consequences (here assumed to be the same as negative effects of labelling) [11]. Similar results are confirmed by our research group in our study population [12].

Haynes et al. recently conducted a large study and found an elevated risk of psychological distress in people aware of the diagnosis of hypertension [13]. However, Haynes used the generic measure GHQ-12. The GHQ12 is a self-administered screening questionnaire, designed for use in consulting settings aimed at detecting those with a diagnosable psychiatric disorder [14]. The purpose of GHQ-12 is not the same as measuring the psychosocial consequences of labelling hypertension.

Furthermore, the use of short-form-12 (SF-12) and SF36 [15] was also proposed to assess the consequences related to hypertension. However, SF-12 and SF-36 are self-administered generic measures for health-related quality of life, and may also fail to measure the psychosocial consequences of labelling hypertension.

Generic measures are instruments designed to be used in broad variety of contexts and to be applicable across conditions and interventions [16]. The downside is that generic measures may lack content validity (coverage and relevance) in terms of specific conditions [10]. Studies have also shown inconsistent psychometric properties of generic instruments when used across different populations [17, 18]. Finally, the use of generic measures becomes problematic if the people who fare least well are also those who find the generic instruments of least relevance [19].

The alternative is to use condition-specific measures, which are instruments that focus on health aspects that are relevant to a specific group of people. Conditionspecific instruments are more sensitive and insure higher content coverage than generic measures [16].

The psychosocial consequences of labelling hypertension seem to be a remarkably frequent patient-reported condition-specific harm [4], and a new questionnaire 
with high content validity and adequate psychometric properties is needed [10]. Methods, which allow accurate measurements of constructs, such as the psychosocial consequences of labelling hypertension, have been developed [20]. One of them is the combination of patient-reported outcomes [9] and Rasch model analysis $[10,21-23]$. In this combination, the development of patient-reported items from qualitative interviews can support the relevance and coverage of the items (content validation) and group the items in different hypothesised domains related to a latent variable. Rasch model analysis can help with determining whether the items grouped in a domain are appropriate indicators and can measure different nuances of the hypothesised latent variable. Such an evidence is necessary to be able to postulate that the score of each item can be added in a sum-score of all the items in a unidimensional scale [24]. Moreover, confirmatory factor analysis (CFA) can be used to confirm the findings of the Rasch model analysis.

Therefore, the aim of this study is to use Rasch model analysis and CFA to screen the pool of items, identify items with sufficient fit to the model and describe the psychometric parameters of the final pool of items.

The tool is not designed to be used in a clinical setting with individual patients to answer questions, such as 'Is my patient experiencing harms of being labelled?' The purpose of this tool is to allow for the measurement of the psychosocial consequences of labelling hypertension in groups of patients and populations. Such measurement is relevant because it can include previously unmeasured harms of being labelled with the diagnosis of hypertension, which can be included in the assessment of the balance between the benefits and harms of medical interventions for preventing CVD in screening for hypertension and for cardiovascular risk assessment [9]. This is a patient-reported outcome measure (PROM) and is supposed to assess the psychosocial consequences of labelling hypertension more accurately than previous measures with GHQ-12, SF-12 and SF-36.

\section{Methods}

We previously developed a Brazilian Portuguese pool of items aiming for the psychosocial consequences of labelling hypertension (Table 1) [12]. That is, we translated items from all versions of the Danish Consequences of Screening (COS) questionnaires [25-28] to Brazilian Portuguese. These COS items, which can be found in all versions of COS, were called 'core' items. Those found in specific versions of COS were called 'disease-specific' items. Then, we conducted single and group interviews with people with hypertension who had low risk of CVD. Subjects selected for our qualitative research had to have a clinically confirmed diagnosis of hypertension with the prescription of antihypertensive medication; we also included only those without comorbidities. These interviews had three main objectives: to test translated items for face and content validity, generate new relevant items to achieve high content validity and to categorise the new items in domains. The items generated on the basis of the interviews were called 'new' items. High content validity was achieved. The result was a set of 132 items divided into 22 domains in two parts. Part I was directed at the general population and encompassed 84 items in 14 domains and 10 single items. Part II was directed only at people diagnosed with hypertension and encompassed 36 items in eight domains and two single items. All items, domains and parts are presented in Table 1. With these methods, we established content relevance and content coverage among Brazilians. To our best knowledge, no other PROM has been developed for the consequences of labelling hypertension.

\section{Sample}

In this study, our target was a sample of the Brazilian population, and the inclusion criteria were: to be older than 18 years old, to be healthy (no self-reported health condition) and to have only hypertension (self-reported hypertension and no other self-reported comorbidity). We collected information about age, gender, ethnic origin, self-reported presence of hypertension, comorbidities, time from diagnosis of hypertension and level of education. A draft questionnaire composed of all the items in the item pool was sent to a target population by using the following strategies. We first used the Survey Monkey ${ }^{\circ}$ Internet-based questionnaire manager to format digital and printed versions of the questionnaire and then distributed it in different media platforms, such as e-mails, WhatsApp ${ }^{\bullet}$ messages and Facebook ${ }^{\circledR}$ invitations. All invitations included a link to the digital questionnaire and could be forwarded to other people. We targeted healthy people and people living with hypertension, but we accepted responses from everyone and used the collected information to separate our target population from the rest afterwards. We also distributed printed versions of the questionnaire among the community healthcare workers around four different primary healthcare clinics. All questionnaires were self-applied. Data were collected in 2017. The responses in the printed versions were transcribed to the data bank by the first author. The draft questionnaire included an informed consent form and sociodemographic items.

\section{Measures}

We selected Rasch model analysis [29] to screen the items and to establish the psychometric properties of this questionnaire because given that it assumes unidimensionality (Rasch models assume that all items reflect 
Table 1 Item pool

\begin{tabular}{|c|c|c|c|c|c|c|}
\hline Part & $\begin{array}{l}\text { Item } \\
\text { number }\end{array}$ & $\begin{array}{l}\text { Included in } \\
\text { final item } \\
\text { set? }\end{array}$ & $\begin{array}{l}\text { Questionnaire } \\
\text { of origin }\end{array}$ & Domain & Brazilian Portuguese version & English ad hoc translation \\
\hline । & 2 & NO & core & Anxiety & Me senti preocupado com meu futuro & I felt worried about my future \\
\hline । & 3 & NO & core & Anxiety & Me senti amedrontado & I felt frightened \\
\hline । & 4 & NO & core & Anxiety & Me senti com medo & I felt scared \\
\hline । & 13 & YES & core & Anxiety & $\begin{array}{l}\text { Me senti emotionalmente fora do meu } \\
\text { normal }\end{array}$ & I felt emotionally out of my normal \\
\hline । & 14 & YES & core & Anxiety & Me senti inquieto & I felt restless \\
\hline । & 15 & YES & core & Anxiety & Me senti nervoso & I felt nervous \\
\hline I & 16 & YES & core & Anxiety & Me senti ansioso & I felt anxious \\
\hline । & 25 & YES & core & Anxiety & Me senti a ponto de entrar em pânico & I felt about to panic \\
\hline । & 29 & YES & disease specific & Anxiety & Me senti em estado de choque & I felt in shock \\
\hline । & 61 & NO & new & Anxiety & Me senti impaciente & I felt impatient \\
\hline । & 93 & NO & core & Anxiety & Me senti agitado & I felt agitated \\
\hline । & 5 & YES & core & Behaviour & Me senti irritado & I felt annoyed \\
\hline 1 & 6 & NO & core & Behaviour & Me senti mais quieto do que o normal & I felt more quiet than usual \\
\hline I & 9 & NO & core & Behaviour & $\begin{array}{l}\text { Me senti com dificuldade de me } \\
\text { concentrar }\end{array}$ & I felt hard to concentrate \\
\hline । & 11 & NO & core & Behaviour & Tive mudanças em meu apetite & I had changes in my appetite \\
\hline । & 18 & NO & core & Behaviour & Me senti mais fechado & I felt introverted \\
\hline । & 22 & YES & core & Behaviour & $\begin{array}{l}\text { Tive dificuldades em realizar meu } \\
\text { trabalho e outras tarefas semelhantes }\end{array}$ & $\begin{array}{l}\text { I had difficulties doing my job and } \\
\text { other similar tasks }\end{array}$ \\
\hline । & 24 & YES & core & Behaviour & $\begin{array}{l}\text { Tive dificuldades em realizar tarefas de } \\
\text { casa }\end{array}$ & I had difficulties doing domestic work \\
\hline । & 30 & NO & new & $\begin{array}{l}\text { Blood pressure } \\
\text { related }\end{array}$ & $\begin{array}{l}\text { Fiquei com medo da pressão alta o } \\
\text { tempo todo na cabeça }\end{array}$ & $\begin{array}{l}\text { I had the fear of high blood pressure } \\
\text { all of the time in the head }\end{array}$ \\
\hline 1 & 57 & NO & new & $\begin{array}{l}\text { Blood pressure } \\
\text { related }\end{array}$ & $\begin{array}{l}\text { Pensei que seria melhor se não } \\
\text { soubesse que tenho pressão alta }\end{array}$ & $\begin{array}{l}\text { I thought it would be better if I didn't } \\
\text { know I have high blood pressure }\end{array}$ \\
\hline । & 90 & NO & new & $\begin{array}{l}\text { Blood pressure } \\
\text { related }\end{array}$ & Tive sintomas de pressão alta & $\begin{array}{l}\text { I had symptoms of high blood } \\
\text { pressure }\end{array}$ \\
\hline | & 37 & YES & disease specific & $\begin{array}{l}\text { Body } \\
\text { Perception }\end{array}$ & Me senti doente & I felt sick \\
\hline । & 38 & YES & disease specific & $\begin{array}{l}\text { Body } \\
\text { Perception }\end{array}$ & $\begin{array}{l}\text { Tive a sensação de que havia algo } \\
\text { errado com meu corpo }\end{array}$ & $\begin{array}{l}\text { I had a feeling something was wrong } \\
\text { with my body }\end{array}$ \\
\hline | & 42 & NO & disease specific & $\begin{array}{l}\text { Body } \\
\text { Perception }\end{array}$ & $\begin{array}{l}\text { Me senti como se meu corpo fosse uma } \\
\text { máquina que não funciona }\end{array}$ & $\begin{array}{l}\text { I felt like my body was a non-working } \\
\text { machine }\end{array}$ \\
\hline । & 46 & YES & disease specific & $\begin{array}{l}\text { Body } \\
\text { Perception }\end{array}$ & $\begin{array}{l}\text { Me senti como um estranho em meu } \\
\text { próprio corpo }\end{array}$ & I felt like a stranger in my own body \\
\hline | & 53 & YES & disease specific & $\begin{array}{l}\text { Body } \\
\text { Perception }\end{array}$ & $\begin{array}{l}\text { Me senti como se qualquer coisa } \\
\text { pudesse me afetar }\end{array}$ & I felt like anything could affect me \\
\hline । & 64 & NO & new & $\begin{array}{l}\text { Body } \\
\text { Perception }\end{array}$ & Senti que não tenho saúde & I felt that I am not healthy \\
\hline | & 69 & NO & new & $\begin{array}{l}\text { Body } \\
\text { Perception }\end{array}$ & Me senti fraco & I felt weak \\
\hline । & 43 & NO & disease specific & Emotional & Me senti azedo & I felt sour \\
\hline । & 44 & NO & disease specific & Emotional & Me senti zangado & I felt angry \\
\hline । & 49 & NO & disease specific & Emotional & Chorei mais do que de costume & I cried more than usual \\
\hline 1 & 63 & NO & new & Emotional & Me senti desequilibrado & I felt unbalanced \\
\hline । & 74 & NO & new & Emotional & Me senti preso & I felt trapped \\
\hline । & 76 & NO & new & Emotional & Me senti orgulhoso & I felt proud \\
\hline
\end{tabular}


Table 1 Item pool (Continued)

\begin{tabular}{|c|c|c|c|c|c|c|}
\hline । & 78 & YES & new & Emotional & Me senti com raiva & I felt angry \\
\hline । & 83 & NO & new & Emotional & Me senti envergonhado & I felt ashamed \\
\hline | & 39 & YES & disease specific & $\begin{array}{l}\text { Fear and } \\
\text { Powerlessness }\end{array}$ & Me senti fora de controle & I felt out of control \\
\hline । & 40 & YES & disease specific & $\begin{array}{l}\text { Fear and } \\
\text { Powerlessness }\end{array}$ & Me senti com o corpo frágil & I felt my body fragile \\
\hline । & 48 & YES & disease specific & $\begin{array}{l}\text { Fear and } \\
\text { Powerlessness }\end{array}$ & Me senti sem forças & I felt strengthless \\
\hline । & 50 & NO & disease specific & $\begin{array}{l}\text { Fear and } \\
\text { Powerlessness }\end{array}$ & Me senti sem sorte & I felt unlucky \\
\hline । & 51 & YES & disease specific & $\begin{array}{l}\text { Fear and } \\
\text { Powerlessness }\end{array}$ & Me senti vulnerável & I felt vulnerable \\
\hline । & 58 & NO & disease specific & $\begin{array}{l}\text { Fear and } \\
\text { Powerlessness }\end{array}$ & Tive medo de fazer esforço físico & I was afraid of doing exercises \\
\hline । & 66 & NO & new & $\begin{array}{l}\text { Fear and } \\
\text { Powerlessness }\end{array}$ & Me senti sem saber o que esperar & I didn't know what to expect \\
\hline । & 73 & NO & new & $\begin{array}{l}\text { Fear and } \\
\text { Powerlessness }\end{array}$ & Me senti com pavor & I felt terrified \\
\hline । & 77 & NO & new & $\begin{array}{l}\text { Fear and } \\
\text { Powerlessness }\end{array}$ & Me senti apreensivo & I felt apprehensive \\
\hline । & 79 & NO & new & $\begin{array}{l}\text { Fear and } \\
\text { Powerlessness }\end{array}$ & Me senti impotente & I felt helpless \\
\hline । & 92 & YES & new & $\begin{array}{l}\text { Fear and } \\
\text { Powerlessness }\end{array}$ & Me senti assustado & I felt scared \\
\hline | & 31 & YES & disease specific & Introvert & Me senti inseguro & I felt insecure \\
\hline I & 32 & YES & disease specific & Introvert & Me senti com pena de mim mesmo & I felt sorry for myself \\
\hline । & 33 & YES & disease specific & Introvert & $\begin{array}{l}\text { Me senti em uma situação } \\
\text { desesperadora }\end{array}$ & I felt in a desperate situation \\
\hline । & 34 & YES & disease specific & Introvert & Fiquei com humor muito variável & I was in a very variable mood \\
\hline | & 54 & YES & disease specific & Lifestyle & Mudei meus hábitos de atividade física & I changed my exercising habits \\
\hline । & 56 & YES & disease specific & Lifestyle & Mudei meus hábitos alimentares & I changed my eating habits \\
\hline । & 72 & YES & new & $\begin{array}{l}\text { Negative } \\
\text { impact on } \\
\text { relations }\end{array}$ & Me senti sendo julgado & I felt that I was being judged \\
\hline | & 75 & YES & new & $\begin{array}{l}\text { Negative } \\
\text { impact on } \\
\text { relations }\end{array}$ & $\begin{array}{l}\text { Me senti sendo forçado a fazer coisas } \\
\text { que não quero }\end{array}$ & $\begin{array}{l}\text { I felt being forced to do things I don't } \\
\text { want to do }\end{array}$ \\
\hline | & 84 & YES & new & $\begin{array}{l}\text { Negative } \\
\text { impact on } \\
\text { relations }\end{array}$ & Me senti controlado pelos outros & I felt that I was controlled by others \\
\hline | & 86 & YES & new & $\begin{array}{l}\text { Negative } \\
\text { impact on } \\
\text { relations }\end{array}$ & Me senti excluído & I felt excluded \\
\hline | & 88 & NO & new & $\begin{array}{l}\text { Neutral impact } \\
\text { on relations }\end{array}$ & Me senti diferente & I felt different \\
\hline | & 41 & NO & disease specific & $\begin{array}{l}\text { Perception of } \\
\text { age }\end{array}$ & Senti que a idade chegou & I felt that old age has come \\
\hline | & 47 & NO & disease specific & $\begin{array}{l}\text { Perception of } \\
\text { age }\end{array}$ & Me senti mais velho do que sou & I felt older than I am \\
\hline | & 85 & NO & new & $\begin{array}{l}\text { Postitive } \\
\text { impact on } \\
\text { relations }\end{array}$ & Me senti apoiado & I felt supported \\
\hline & 87 & NO & new & $\begin{array}{l}\text { Postitive } \\
\text { impact on } \\
\text { relations }\end{array}$ & Me senti cuidado & I felt being cared for \\
\hline
\end{tabular}


Table 1 Item pool (Continued)

\begin{tabular}{|c|c|c|c|c|c|c|}
\hline I & 89 & NO & new & $\begin{array}{l}\text { Postitive } \\
\text { impact on } \\
\text { relations }\end{array}$ & Me senti importante & I felt important \\
\hline । & 65 & NO & new & $\begin{array}{l}\text { Results of } \\
\text { diagnosis }\end{array}$ & Me senti em dúvida & I felt in doubt \\
\hline । & 80 & NO & new & $\begin{array}{l}\text { Results of } \\
\text { diagnosis }\end{array}$ & Me senti surpreso & I felt surprised \\
\hline । & 1 & YES & core & $\begin{array}{l}\text { Sense of } \\
\text { dejection }\end{array}$ & Me senti preocupado & I felt worried \\
\hline । & 10 & YES & core & $\begin{array}{l}\text { Sense of } \\
\text { dejection }\end{array}$ & $\begin{array}{l}\text { Me senti com a sensação de que o } \\
\text { tempo não passava }\end{array}$ & I felt that time was not passing \\
\hline । & 12 & YES & core & $\begin{array}{l}\text { Sense of } \\
\text { dejection }\end{array}$ & Me senti triste & I felt sad \\
\hline । & 19 & YES & core & $\begin{array}{l}\text { Sense of } \\
\text { dejection }\end{array}$ & Me senti sem iniciativa & I felt without initiative \\
\hline । & 20 & NO & core & $\begin{array}{l}\text { Sense of } \\
\text { dejection }\end{array}$ & Me senti sem vontade & I felt unwilling \\
\hline I & 21 & NO & core & $\begin{array}{l}\text { Sense of } \\
\text { dejection }\end{array}$ & Me senti deprimido & I felt depressed \\
\hline । & 62 & YES & new & $\begin{array}{l}\text { Sense of } \\
\text { dejection }\end{array}$ & Me senti culpado & I felt guilty \\
\hline । & 67 & YES & new & $\begin{array}{l}\text { Sense of } \\
\text { dejection }\end{array}$ & Me senti desmotivado & I felt unmotivated \\
\hline । & 68 & YES & new & $\begin{array}{l}\text { Sense of } \\
\text { dejection }\end{array}$ & Me senti desestimulado & I felt discouraged \\
\hline | & 70 & NO & new & $\begin{array}{l}\text { Sense of } \\
\text { dejection }\end{array}$ & Me senti frustrado & I felt frustrated \\
\hline । & 71 & YES & new & $\begin{array}{l}\text { Sense of } \\
\text { dejection }\end{array}$ & Me senti indiferente & I felt indifferent \\
\hline | & 82 & YES & new & $\begin{array}{l}\text { Sense of } \\
\text { dejection }\end{array}$ & Me senti chateado & I felt upset \\
\hline । & 91 & NO & new & $\begin{array}{l}\text { Sense of } \\
\text { dejection }\end{array}$ & $\begin{array}{l}\text { Me senti culpado por não cuidar de } \\
\text { mim mesmo como deveria }\end{array}$ & $\begin{array}{l}\text { I felt guilty for not taking care of } \\
\text { myself as I should }\end{array}$ \\
\hline | & 94 & YES & core & $\begin{array}{l}\text { Sense of } \\
\text { dejection }\end{array}$ & Me senti incomodado & I felt bothered \\
\hline । & 27 & YES & core & Sexual & Tive menos desejo sexual & I had less sexual desire \\
\hline | & 59 & YES & disease specific & Sexual & $\begin{array}{l}\text { Me senti insatisfeito com minha vida } \\
\text { sexual }\end{array}$ & I felt dissatisfied with my sex life \\
\hline । & 8 & YES & core & Single Items & $\begin{array}{l}\text { Fuji dos meus pensamentos me } \\
\text { ocupando com tarefas práticas do dia-a- } \\
\text { dia }\end{array}$ & $\begin{array}{l}\text { I ran away from my thoughts, busy } \\
\text { with day-to-day practical tasks }\end{array}$ \\
\hline । & 28 & YES & core & Single Items & Dias faltados no trabalho & Days missed at work \\
\hline | & 35 & YES & disease specific & Single Items & $\begin{array}{l}\text { Me senti mais cansado do que de } \\
\text { costume }\end{array}$ & I felt more tired than usual \\
\hline | & 36 & YES & disease specific & Single Items & Guardei meus pensamentos só pra mim & I kept my thoughts just for myself \\
\hline । & 45 & YES & disease specific & Single Items & Me senti como se estivesse no vazio & I felt like I was in the void \\
\hline | & 52 & YES & disease specific & Single Items & Me senti fragilizado & I felt weak \\
\hline | & 55 & YES & disease specific & Single Items & Pensei na morte & I thought about death \\
\hline | & 60 & YES & new & Single Items & Pensei na minha fé & I thought of my faith \\
\hline | & 81 & YES & new & Single Items & Me senti tranquilo & I felt calm \\
\hline | & 95 & YES & new & Single Items & Você tem pressão alta? & Do you have a high blood pressure? \\
\hline 1 & 7 & NO & core & Sleep & Dormi mal à noite & I slept badly at night \\
\hline & 17 & NO & core & Sleep & Tive dificuldade de pegar no sono & I had difficulty falling asleep \\
\hline
\end{tabular}


Table 1 Item pool (Continued)

\begin{tabular}{|c|c|c|c|c|c|c|}
\hline । & 23 & NO & core & Sleep & Acordei cedo demais & I woke up too early \\
\hline । & 26 & NO & core & Sleep & $\begin{array}{l}\text { Passei a maior parte do tempo } \\
\text { acordado }\end{array}$ & I spent most of the time awake \\
\hline Part & $\begin{array}{l}\text { Item } \\
\text { number }\end{array}$ & $\begin{array}{l}\text { Included in } \\
\text { final version }\end{array}$ & $\begin{array}{l}\text { Questionnaire } \\
\text { of origin }\end{array}$ & Domain & Brazilian Portuguese version & English ad hoc translation \\
\hline$\|$ & 108 & YES & disease specific & Empathy & $\begin{array}{l}\text { meu sentimento de responsabilidade } \\
\text { pela minha família ficou }\end{array}$ & $\begin{array}{l}\text { my sense of responsibility for my } \\
\text { family became... }\end{array}$ \\
\hline$\|$ & 111 & YES & disease specific & Empathy & $\begin{array}{l}\text { minha compreensão dos problemas } \\
\text { alheios ficou }\end{array}$ & $\begin{array}{l}\text { my understanding of other people's } \\
\text { problems became ... }\end{array}$ \\
\hline$\|$ & 113 & YES & disease specific & Empathy & $\begin{array}{l}\text { a minha capacidade de ouvir problemas } \\
\text { alheios ficou }\end{array}$ & $\begin{array}{l}\text { my ability to hear other people's } \\
\text { problems became... }\end{array}$ \\
\hline$\|$ & 96 & YES & core & $\begin{array}{l}\text { Existential } \\
\text { values }\end{array}$ & eu fiquei pensando na vida & I kept thinking about life... \\
\hline$\|$ & 97 & YES & core & $\begin{array}{l}\text { Existential } \\
\text { values }\end{array}$ & minha alegria de viver ficou & my joy of living became ... \\
\hline$\|$ & 103 & YES & core & $\begin{array}{l}\text { Existential } \\
\text { values }\end{array}$ & a minha visão do futuro ficou & my vision of the future became ... \\
\hline$\|$ & 104 & YES & core & $\begin{array}{l}\text { Existential } \\
\text { values }\end{array}$ & a minha sensação de bem-estar ficou & my sense of well-being became ... \\
\hline$\|$ & 105 & YES & core & $\begin{array}{l}\text { Existential } \\
\text { values }\end{array}$ & a minha percepção sobre a vida ficou & my perception of life became ... \\
\hline$\|$ & 106 & YES & core & $\begin{array}{l}\text { Existential } \\
\text { values }\end{array}$ & o valor que dou a vida ficou & the value I give in life became ... \\
\hline$\|$ & 125 & YES & new & $\begin{array}{l}\text { Existential } \\
\text { values }\end{array}$ & $\begin{array}{l}\text { me sinto como se não fosse mais } \\
\text { normal }\end{array}$ & I feel like I'm not normal anymore... \\
\hline$\|$ & 126 & YES & new & $\begin{array}{l}\text { Existential } \\
\text { values }\end{array}$ & $\begin{array}{l}\text { me sinto como se não fosse mais o } \\
\text { mesmo }\end{array}$ & I feel like I'm not the same anymore... \\
\hline$\|$ & 132 & NO & new & $\begin{array}{l}\text { Hypertension } \\
\text { related }\end{array}$ & $\begin{array}{l}\text { minha ansiedade com relação a pressão } \\
\text { alta ficou }\end{array}$ & $\begin{array}{l}\text { my anxiety about high blood pressure } \\
\text { got... }\end{array}$ \\
\hline$\|$ & 133 & NO & new & $\begin{array}{l}\text { Hypertension } \\
\text { related }\end{array}$ & penso que eu não tenho pressão alta & $\begin{array}{l}\text { I think I don't have high blood } \\
\text { pressure... }\end{array}$ \\
\hline$\|$ & 107 & YES & disease specific & Impulsive & a minha energia ficou & my energy became ... \\
\hline$\|$ & 109 & YES & disease specific & Impulsive & tenho aproveitado a vida & I have enjoyed life... \\
\hline$\|$ & 112 & YES & disease specific & Impulsive & me sinto impulsivo & I feel impulsive... \\
\hline$\|$ & 114 & YES & disease specific & Impulsive & $\begin{array}{l}\text { a minha vontade de me envolver com } \\
\text { algo novo ficou }\end{array}$ & $\begin{array}{l}\text { my desire to get involved with } \\
\text { something new became ... }\end{array}$ \\
\hline$\|$ & 115 & YES & disease specific & Impulsive & $\begin{array}{l}\text { a minha vontade de me envolver com } \\
\text { algo arriscado ficou }\end{array}$ & $\begin{array}{l}\text { my desire to get involved with } \\
\text { something risky got ... }\end{array}$ \\
\hline$\|$ & 116 & YES & disease specific & Impulsive & $\begin{array}{l}\text { tenho feito coisas que utrapassam meus } \\
\text { limites }\end{array}$ & $\begin{array}{l}\text { I've been doing things that push my } \\
\text { limits... }\end{array}$ \\
\hline$\|$ & 117 & YES & new & Patient Role & frequento consultas médicas & I go to doctor's appointments... \\
\hline$\|$ & 118 & YES & new & Patient Role & faço exames & I do laboratory tests... \\
\hline$\|$ & 119 & NO & new & Patient Role & me sinto fazendo mal para mim mesmo & I feel bad for myself... \\
\hline$\|$ & 120 & YES & new & Patient Role & $\begin{array}{l}\text { me sinto com dificuldades em seguir } \\
\text { orientações médicas }\end{array}$ & $\begin{array}{l}\text { I have difficulty following medical } \\
\text { advices... }\end{array}$ \\
\hline$\|$ & 121 & YES & new & Patient Role & me sinto cuidando de mim mesmo & I feel taking care of myself... \\
\hline$\|$ & 122 & YES & new & Patient Role & tomo medicamentos & I take medicines... \\
\hline$\|$ & 123 & YES & new & Patient Role & me sinto dependente de remédios & I feel dependent on medicines... \\
\hline$\|$ & 124 & YES & new & Patient Role & $\begin{array}{l}\text { me sinto confiante em orientações } \\
\text { médicas }\end{array}$ & I feel confident in medical advice... \\
\hline$\|$ & 99 & YES & core & $\begin{array}{l}\text { Personal } \\
\text { Relations }\end{array}$ & $\begin{array}{l}\text { a minha relação com a minha família } \\
\text { ficou }\end{array}$ & $\begin{array}{l}\text { my relationship with my family } \\
\text { became... }\end{array}$ \\
\hline$\|$ & 100 & YES & core & Personal & a minha relação com meus amigos ficou & my relationship with my friends \\
\hline
\end{tabular}


Table 1 Item pool (Continued)

\begin{tabular}{|c|c|c|c|c|c|c|}
\hline & & & & Relations & & became ... \\
\hline$\|$ & 101 & YES & core & $\begin{array}{l}\text { Personal } \\
\text { Relations }\end{array}$ & $\begin{array}{l}\text { a minha relação com outras pessoas } \\
\text { ficou }\end{array}$ & $\begin{array}{l}\text { my relationship with other people } \\
\text { became... }\end{array}$ \\
\hline$\|$ & 127 & YES & new & $\begin{array}{l}\text { Preoccupation } \\
\text { with health }\end{array}$ & $\begin{array}{l}\text { me sinto preocupado com sintomas de } \\
\text { pressão alta }\end{array}$ & $\begin{array}{l}\text { I feel worried about symptoms of } \\
\text { high blood pressure... }\end{array}$ \\
\hline$\|$ & 128 & YES & new & $\begin{array}{l}\text { Preoccupation } \\
\text { with health }\end{array}$ & $\begin{array}{l}\text { me sinto preocupado com meus } \\
\text { hábitos e estilo de vida }\end{array}$ & $\begin{array}{l}\text { I feel worried about my habits and } \\
\text { lifestyle... }\end{array}$ \\
\hline$\|$ & 129 & YES & new & $\begin{array}{l}\text { Preoccupation } \\
\text { with health }\end{array}$ & $\begin{array}{l}\text { me sinto preocupado com os } \\
\text { tratamentos }\end{array}$ & I feel worried about the treatments... \\
\hline$\|$ & 98 & NO & core & Relaxed/Calm & me senti tranquilo & I felt tranquil... \\
\hline$\|$ & 102 & NO & core & Relaxed/Calm & me senti calmo & I felt calm... \\
\hline$\|$ & 110 & NO & core & Relaxed/Calm & me sinto aliviado & I feel relieved... \\
\hline$\|$ & 130 & YES & new & Single Items & meu desempenho no trabalho ficou & my work performance became ... \\
\hline ॥ & 131 & YES & new & Single Items & minha prática sexual ficou & my sexual practice became ... \\
\hline
\end{tabular}

all items in the item pool in Brazilian Portuguese and the English ad hoc translations. Domains of each part are in alphabetical order

an underlying construct), it allows to investigate the fit of the items to a hypothesised dimension and how these items are interrelated and ordered on a latent continuum; thus, it supports the addition of the raw scores of items into a single score [30].

We referred to the qualitative material whenever an item did not fit the model and tried to understand why they did not fit. We aimed at two features of the Rasch models during the psychometric analysis: local response dependence (LD) [31] and differential item functioning (DIF) [32]. LD occurs when two items capture unique common information independently from what is supposed to be measured by the item set. That is, the answer of an item should not influence the answer of another item. Meanwhile, DIF occurs when the expected responses of individuals with the same level (but belong to different groups defined by an external factor) for a measured construct differ. That is, an external factor should not influence the answer of an item [33]. We included age (defined as age above or below 40), gender (male or female), ethnicity and the presence or absence of hypertension in our analysis.

To provide the measurement of psychosocial consequences consistent with Rasch measurement theory, the subscales calculated from the data collected for psychometric analysis should fit a graphical Rasch model (GRM) [34-36]. The overall model fit was assessed using the Andersen conditional likelihood ratio test [37] and the individual item fit was evaluated by comparing observed and expected item-rest score associations [22].

We also evaluated item fit graphically by dividing the sample into five score groups. For each item, we plotted the item mean score in each interval and compared all the scores to $95 \%$ confidence regions of the model expectations. For each item, the observed mean score in each class interval was plotted as a line together with a shaded area that indicates the 95\% confidence region of the model expectations. Thus, when curves are contained in the shaded area, the observed data match the model expectations and thus indicate item fit.

The following was the modelling strategy:

(i) evaluating the fit of the COS core items in their previously identified domains to the Rasch models;

(ii) evaluating the fit of the COS core items to a GRM derived using item screening procedure, assessing the issues of COS core problematic items and removing them from the subscale;

(iii) adding COS disease-specific items to the subscale;

(iv) evaluating the fit of the COS disease-specific (+ COS core) items to the GRM, assessing the issues of COS disease-specific problematic items and removing them from the subscale;

(v) adding new items to the subscale;

(vi) evaluating the fit of the new items (new + COS items) to GRM, assessing the issues of problematic items and removing them from the subscale;

(vii)if possible, confirming the dimensionality of the derived subscales by using CFA;

(viiìvaluating reliability using Cronbach's coefficient alpha.

After the Rasch model analysis, we used in each subscale CFA to confirm the fit indices and Cronbach's alpha to test reliability.. In CFA and Cronbach's alpha, missing data were excluded, and only complete responses were assessed. We used the evidence of local dependence found in the Rasch model analysis to indicate the correlated error terms in the CFA model. CFA was used only for subscales with four or more items after the Rasch model analysis. Rasch model analysis was conducted using the 
computer programme DIGRAM [38]. CFA and Cronbach's alpha were conducted in STATA.

The null hypothesis of the statistical tests in the Rasch model analysis was that the model fits. We adjusted $p$ values by using the Benjamini-Hochberg [39] procedure to control the false discovery rate at $5 \%$ and thus took values above 0.05 as cut-off values for model fit. In CFA, the cut-off values were 0.06 for RMSEA and 0.95 for CFI [40]. Values above 0.70 for Cronbach's coefficient alpha were considered adequate [41].

\section{Results}

\section{Sample}

We collected 1118 responses. After the exclusion of 319 informants with comorbidities, the validation sample consisted of 798 respondents living in all five Brazilian regions and 26 states that were recruited via different media platforms in the following proportion: $47.1 \%$ responded via the WhatsApp link, 36.7\% responded via the Facebook ${ }^{\circ}$ link, 9.7\% responded to the email invitation and $6,4 \%$ responded to the paper version.

Out of the 798 respondents, 285 (35.7\%) were diagnosed with hypertension, 597 (74.8\%) were female, 460 (57.6\%) were over 40 years old, 566 (70.9\%) were Caucasian and 204 (25.5\%) had less than 11 years of education. All 798 respondents completed Part I, whereas 285 respondents with hypertension completed Part II. (Table 2. Population characteristics).

Forty-four $(46.8 \%)$ of the 94 items in Part I were rejected; thus, 41 items in 10 dimensions (Fig. 1): 'anxiety', 'behaviour', 'body perception', 'emotional', 'fear and powerlessness', 'introvert', 'lifestyle', 'negative relations', 'sense of dejection' and 'sexual' and 9 single items remained.
Six (15.7\%) of the 38 items in Part II were rejected; thus 30 items in five dimensions (Fig. 1): 'empathy', 'existential values', 'impulsive', 'patient role + preoccupation with health' and 'personal relations' and two single items remained. A 71-item questionnaire with two parts was yielded, with 15 dimensions and 11 single items. The main reason for the exclusion of items was $65 \%$ of the cases failed to fit, followed by $30 \%$ of DIF cases. All DIF cases were found in the items of Part I. The main variable responsible for DIF was the presence of hypertension found in seven of the 17 items that were excluded for this reason. Age was responsible for DIF in five items, gender in three and ethnicity in two items. (Table 3. Rejected items and reasons for the exclusion).

The graphical model check showed that as the domain score increased, items' mean scores also increased, indicating that all items within a domain measure the same construct. All plots are presented in the Additional file 1.

\section{Measures \\ Part I}

Rasch model analysis We had 10 single items for Part I that were derived from the content validation study. Based on the qualitative assessment of the item pool, we hypothesised that six of them $(35,36,45,52,75$ and 81$)$ could be tested in the following domains-35 in 'body perception', 36 in 'emotional' and in 'introvert', 81 in 'emotional', 52 in 'emotional' and in 'fear and powerlessness', 45 in 'perception of age' and 75 in 'negative relations.' Item 75 found fit in the domain, whereas the five other items were rejected in the tested domains. The

Table 2 Population characteristics

\begin{tabular}{|c|c|c|c|c|}
\hline Characteristics & \multicolumn{2}{|c|}{ no hypertension $n=513$} & \multicolumn{2}{|c|}{ hypertension $n=285$} \\
\hline mean age, years & \multicolumn{2}{|c|}{$39.4(18-73)$} & \multicolumn{2}{|c|}{$53.0(20-85)$} \\
\hline mean education, years & \multicolumn{2}{|c|}{$17.6(0-32)$} & \multicolumn{2}{|c|}{$11.7(0-30)$} \\
\hline mean time from diagnosis, years & \multicolumn{2}{|l|}{-} & \multicolumn{2}{|c|}{$10.1(0.1-40)$} \\
\hline \multicolumn{5}{|l|}{ Gender } \\
\hline male & 138 & $27 \%$ & 63 & $22 \%$ \\
\hline female & 375 & $73 \%$ & 222 & $78 \%$ \\
\hline \multicolumn{5}{|l|}{ Ethnic origin } \\
\hline afro + multi & 124 & $24 \%$ & 104 & $36 \%$ \\
\hline caucaso + asian & 386 & $75 \%$ & 180 & $63 \%$ \\
\hline \multicolumn{5}{|l|}{ Response media } \\
\hline e-mail & 67 & $13 \%$ & 11 & $4 \%$ \\
\hline Facebook & 140 & $27 \%$ & 153 & $54 \%$ \\
\hline printed & 3 & $1 \%$ & 48 & $17 \%$ \\
\hline WhatsApp & 303 & $59 \%$ & 73 & $26 \%$ \\
\hline
\end{tabular}

mean age, education, and time from diagnosis. Frequency of gender, ethnicity, and response media in the tow groups: 'no hypertension' and 'hypertension' 


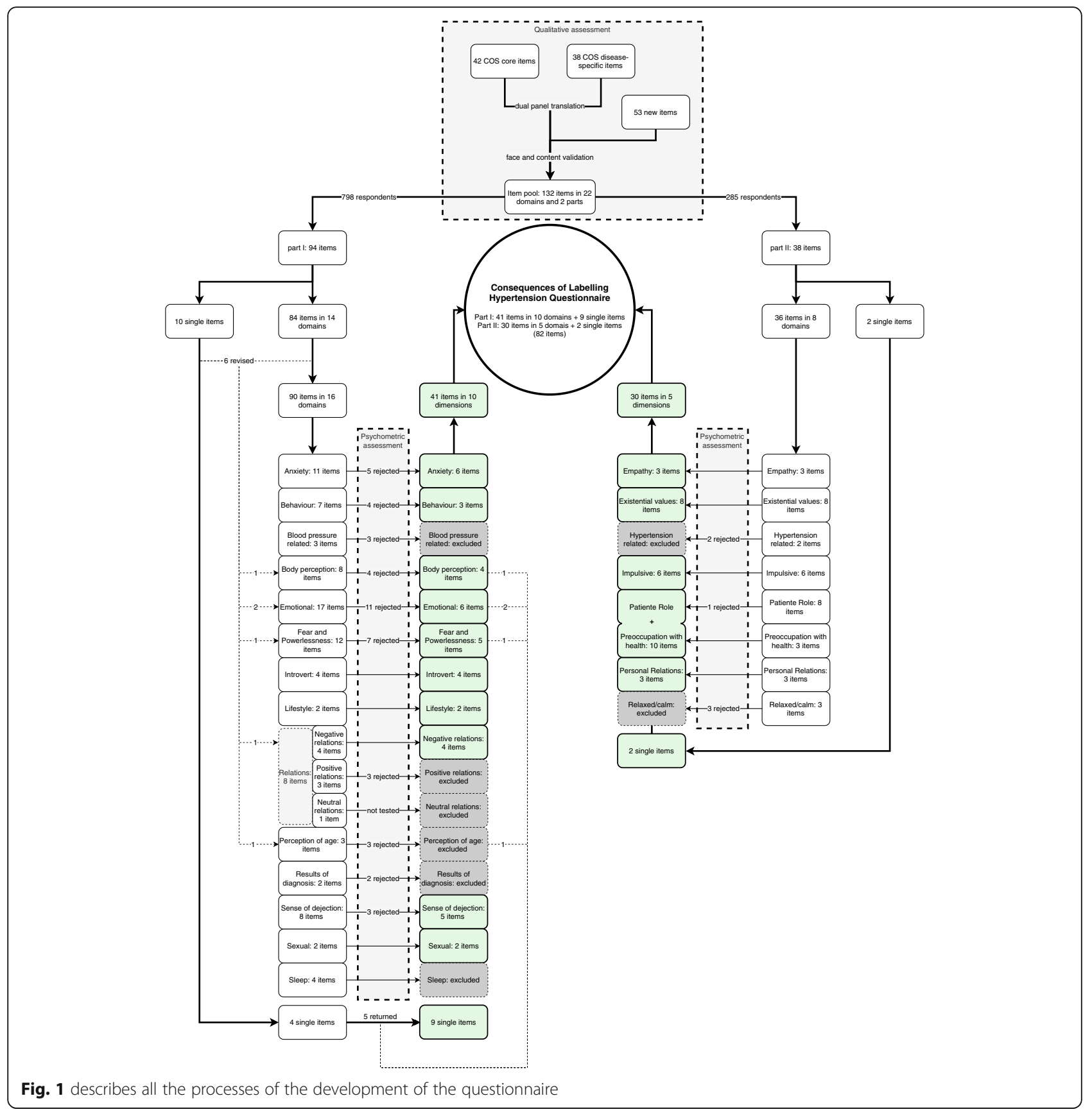

nine items that failed to find a place in a subscale were kept as single items.

Twenty-eight items derived from COS core were tested in five different dimensions: 'anxiety', 'behaviour', 'sense of dejection', 'sexual' and 'sleep.' The 'sleep' dimension, which was composed only by core items, did not fit the Rasch model analysis no matter the combination of the four sleep items. Fourteen of the remaining 24 items fitted the four other respective dimensions.

Twenty-eight items derived from other COS disease-specific questionnaires were tested in eight different dimensions: 'anxiety', 'body perception', 'emotional', 'fear and powerlessness', 'introvert', 'lifestyle', 'perception of age' and 'sexual.' Four of these dimensions had altogether 12 items rejected: 'body perception', 'emotional', 'fear and powerlessness' and 'perception of age', whereas 16 items fitted the respective domains.

Thirty-four new items were tested in nine different dimensions. Four of these were newly created dimensions: 'blood pressure related', 'relations negative', 'relations positive' and 'results of the diagnosis.' The five other 
Table 3 Rejected items and reasons for the exclusion

\begin{tabular}{|c|c|c|c|c|}
\hline Part & Domain & $\mathrm{Q}$ of origin & item number & reason for exclusion \\
\hline \multirow[t]{44}{*}{ । } & Anxiety & core & 2 & no fit \\
\hline & & core & 3 & no fit \\
\hline & & core & 4 & no fit \\
\hline & & core & 93 & too many missing responses \\
\hline & & new & 61 & no fit \\
\hline & Behaviour & core & 6 & DIF \\
\hline & & core & 9 & DIF \\
\hline & & core & 11 & DIF \\
\hline & & core & 18 & DIF \\
\hline & Blood pressure related & new & 30 & DIF \\
\hline & & new & 57 & DIF \\
\hline & & new & 90 & DIF \\
\hline & Body Perception & disease specific & 35 & DIF \\
\hline & & disease specific & 42 & DIF \\
\hline & & new & 64 & DIF \\
\hline & & new & 69 & no fit \\
\hline & Emotional & disease specific & 43 & no fit \\
\hline & & disease specific & 44 & no fit \\
\hline & & disease specific & 49 & DIF \\
\hline & & new & 63 & no fit \\
\hline & & new & 74 & no fit \\
\hline & & new & 76 & no fit \\
\hline & & new & 83 & DIF \\
\hline & & new & 70 & no fit \\
\hline & & new & 81 & no fit \\
\hline & & disease specific & 36 & no fit \\
\hline & & disease specific & 52 & no fit \\
\hline & Fear and Powerlessness & disease specific & 50 & DIF \\
\hline & & disease specific & 58 & DIF \\
\hline & & new & 66 & no fit \\
\hline & & new & 73 & no fit \\
\hline & & new & 77 & no fit \\
\hline & & new & 79 & no fit \\
\hline & & disease specific & 52 & no fit \\
\hline & Introvert & disease specific & 36 & DIF \\
\hline & Perception of age & disease specific & 41 & no fit \\
\hline & & disease specific & 47 & no fit \\
\hline & & disease specific & 45 & no fit \\
\hline & Positive relations & new & 85 & no fit \\
\hline & & new & 87 & no fit \\
\hline & & new & 89 & no fit \\
\hline & Results of diagnosis & new & 65 & no fit \\
\hline & & new & 80 & no fit \\
\hline & Sense of dejection & core & 20 & 19 fits better than 20 \\
\hline
\end{tabular}


Table 3 Rejected items and reasons for the exclusion (Continued)

\begin{tabular}{|c|c|c|c|c|}
\hline Part & Domain & $\mathrm{Q}$ of origin & item number & reason for exclusion \\
\hline & & core & 21 & DIF \\
\hline & & new & 91 & DIF \\
\hline & Sleep & core & 7 & no fit \\
\hline & & core & 17 & no fit \\
\hline & & core & 23 & no fit \\
\hline & & core & 26 & no fit \\
\hline & Social Relations & new & 88 & neutral \\
\hline \multirow[t]{6}{*}{$\|$} & Hypertension related & new & 132 & no fit \\
\hline & & new & 133 & no fit \\
\hline & Patient Role & new & 119 & no fit \\
\hline & Relaxed/Calm & core & 98 & no fit \\
\hline & & core & 102 & no fit \\
\hline & & core & 110 & no fit \\
\hline
\end{tabular}

dimensions that had new items tested were 'anxiety', 'body perception', 'emotional', 'fear and powerlessness' and 'sense of dejection.' Twenty-three new items were rejected, and 11 were accepted in the tested dimensions.

Eight items comprised the 'social relations' dimension (72, 75, 84, 85, 86, 87, 88 and 89). The first analysis that included all items suggested two subscales with opposite relational effects and one neutral item. We then excluded the neutral item (88) and split the items in two dimensions: 'positive relations' with Items 85,87 and 89 and 'negative relations' with the remaining Items 72,75 , 84 and 86 . The 'positive relations' dimension failed to find fit, but the 'negative relations' found fit with DIF with age for Item 72 (being judged): those over 40 years old consistently scored lower than those under 40 who have the same total score.

Items 27 and 59 in the 'sexual' dimension showed DIF with gender. Women consistently scored higher on Item 27 and lower on Item 59 compared with men.

In the 'emotional' dimension, item pairs 67/68 and 78/ 82 had LD. In the 'anxiety' dimension, item pairs $25 / 29$ also had LD. The same was revealed for item pair $22 / 24$ in 'behaviour', item pairs $37 / 38$ and $46 / 53$ in 'body perception', item pair 75/84 in 'negative relations' and finally item pairs $10 / 19$ and $12 / 19$ in 'sense of dejection.' In all these cases, these pairs fitted the subscales.

Items 3 and 4 were different versions of the same item, and we included only one of them at a time in the 'anxiety' dimension. We began with two versions of the subscale, each with either Item 3 or 4 and then tried to add new items. However, in both versions, these items misfit and were excluded from the final version of the subscale. Items 19 and 20 were also two different versions of the original item. The 'sense of dejection' dimension showed good fit with Item 19.
The following domains had no items selected and were excluded from the final questionnaire: 'blood pressure related' with three items, 'perception of age' with two items, 'positive relations' with three items, 'results of the diagnosis' with two items, 'sleep' with four items and 'neutral relations' with one item. The results of the Rasch model analysis are shown in Table 4 with the selected set of items for each subscale.

CFA and reliability Table 4 presents the CFA parameters for Part I. Two subscales had an RMSEA above 0.06 ('anxiety' and 'fear and powerlessness'), whereas none had CFI below 0.95 .

All 10 accepted subscales were tested for internal consistency with Cronbach's alpha coefficients. Two subscales, 'lifestyle' and 'sense of dejection', had alpha values below 0.7 .

\section{Part II}

Rasch model analysis Twelve items derived from COS core were tested in three different dimensions: 'existential values', 'personal relations' and 'relaxed/calm.' The 'relaxed/calm' items neither fitted the Rasch model analysis nor formed a scale. All other core items found fit. Local dependence was observed between Items 103 and 104.

Nine items derived from COS disease-specific items were tested in two different dimensions: 'empathy' and 'impulsive'. All items were accepted. Local dependence was found between Items 111 and 113 .

Fifteen new items were tested in four different dimensions: 'existential values', 'hypertension related', 'patient role' and 'preoccupation with health.' The 'existential values' dimension was the only one that had items from 
Table 4 Selected items: part I

\begin{tabular}{|c|c|c|c|c|c|c|c|c|c|}
\hline & \multirow[b]{2}{*}{ Scale } & \multicolumn{5}{|c|}{ Rasch model analysis results } & \multicolumn{2}{|c|}{$\begin{array}{l}\text { Confirmatory } \\
\text { Factor } \\
\text { Analysis } \\
\text { results }\end{array}$} & \multirow{2}{*}{$\begin{array}{l}\text { Internal } \\
\text { consistency }\end{array}$} \\
\hline & & $\begin{array}{l}\text { number of items } \\
\text { tested }\end{array}$ & $\begin{array}{l}\text { number of items } \\
\text { selected }\end{array}$ & $\begin{array}{l}\text { selected item's } \\
\text { number }\end{array}$ & CML & $p$ & rmsea & CFI & \\
\hline \multirow{41}{*}{$\begin{array}{l}\text { Part } \\
\text { I }\end{array}$} & \multirow[t]{6}{*}{ Anxiety } & \multirow[t]{6}{*}{11} & \multirow[t]{6}{*}{6} & 13 & \multirow[t]{6}{*}{32.2} & \multirow[t]{6}{*}{0.046} & \multirow[t]{6}{*}{0.062} & \multirow[t]{6}{*}{0.986} & \multirow[t]{6}{*}{0.839} \\
\hline & & & & 14 & & & & & \\
\hline & & & & 15 & & & & & \\
\hline & & & & 16 & & & & & \\
\hline & & & & 25 & & & & & \\
\hline & & & & 29 & & & & & \\
\hline & \multirow[t]{3}{*}{ Behaviour } & \multirow[t]{3}{*}{7} & \multirow[t]{3}{*}{3} & 5 & \multirow[t]{3}{*}{18.9} & \multirow[t]{3}{*}{0.332} & & & \multirow[t]{3}{*}{0.708} \\
\hline & & & & 22 & & & & & \\
\hline & & & & 24 & & & & & \\
\hline & \multirow[t]{4}{*}{ Body perception } & \multirow[t]{4}{*}{8} & \multirow[t]{4}{*}{4} & 37 & \multirow[t]{4}{*}{14.7} & 0.987 & 0,000 & 1000 & 0.806 \\
\hline & & & & 38 & & & & & \\
\hline & & & & 46 & & & & & \\
\hline & & & & 53 & & & & & \\
\hline & Emotional & 17 & 6 & 62 & 54.2 & 0.015 & 0.049 & 0.987 & 0.851 \\
\hline & & & & 67 & & & & & \\
\hline & & & & 68 & & & & & \\
\hline & & & & 71 & & & & & \\
\hline & & & & 78 & & & & & \\
\hline & & & & 82 & & & & & \\
\hline & Fear and & 12 & 5 & 39 & 34.2 & 0.063 & 0.090 & 0.978 & 0.837 \\
\hline & & & & 40 & & & & & \\
\hline & & & & 48 & & & & & \\
\hline & & & & 51 & & & & & \\
\hline & & & & 92 & & & & & \\
\hline & Introvert & 5 & 4 & 31 & 17.1 & 0.106 & 0.047 & 0.997 & 0.804 \\
\hline & & & & 32 & & & & & \\
\hline & & & & 33 & & & & & \\
\hline & & & & 34 & & & & & \\
\hline & Lifestyle & 2 & 2 & 54 & 5 & 0.418 & & & 0.599 \\
\hline & & & & 56 & & & & & \\
\hline & Negative relations & 4 & 4 & 72 & 10.2 & 0.513 & 0,000 & 1000 & 0.777 \\
\hline & & & & 75 & & & & & \\
\hline & & & & 84 & & & & & \\
\hline & & & & 86 & & & & & \\
\hline & Sense of dejection & 8 & 5 & 1 & 39.4 & 0.172 & 0.054 & 0.988 & 0.682 \\
\hline & & & & 10 & & & & & \\
\hline & & & & 12 & & & & & \\
\hline & & & & 19 & & & & & \\
\hline & & & & 94 & & & & & \\
\hline & Sexual & 2 & 2 & 27 & 11.3 & 0.045 & & & 0.722 \\
\hline & & & & 59 & & & & & \\
\hline
\end{tabular}


more than one origin tested (core and new). Three of the items were rejected: one in the 'patient role' dimension (Item 119) and two in the 'hypertension related' dimension (Items 132 and 133). We had two single items for Part II, both new items.

The qualitative assessment of the items of 'patient role' and 'preoccupation with health' suggested that they could be all part of a combined scale called the 'patient role + preoccupation with health' subscale. The Rasch model analysis, where both scales were combined, had a nice fit with no DIF, resulting in a new 10-item subscale: Items $117,118,120,121,122$, 123, 124, 127, 128 and 129.

The following domains had no items selected and were excluded from the final questionnaire: 'hypertension related' with two items and 'relaxed/calm' with three items.

CFA and reliability Table 5 presents the CFA parameters for Part II. Two subscales had RMSEA above 0.06 ('impulsive' and 'patient role + preoccupation with health'), whereas none had CFI below 0.95 .

Table 5 Selected items: part ॥

\begin{tabular}{|c|c|c|c|c|c|c|c|c|c|}
\hline & & Rasch model analys & results & & & & $\begin{array}{l}\text { Confir } \\
\text { Factor } \\
\text { Analys } \\
\text { results }\end{array}$ & natory & $\begin{array}{l}\text { Internal } \\
\text { consistency }\end{array}$ \\
\hline & Scale & $\begin{array}{l}\text { number of items in } \\
\text { the pool }\end{array}$ & $\begin{array}{l}\text { number of items } \\
\text { selected }\end{array}$ & $\begin{array}{l}\text { selected item's } \\
\text { number }\end{array}$ & CML & $\mathbf{p}$ & rmsea & CFI & Cronbach-alfa \\
\hline Part & Empathy & 3 & 3 & 108 & 6.6 & 0.712 & & & 0.733 \\
\hline & & & & 111 & & & & & \\
\hline & & & & 113 & & & & & \\
\hline & Existential values & 8 & 8 & 96 & 31.0 & 0.124 & 0.054 & 0.982 & 0.860 \\
\hline & & & & 97 & & & & & \\
\hline & & & & 103 & & & & & \\
\hline & & & & 104 & & & & & \\
\hline & & & & 105 & & & & & \\
\hline & & & & 106 & & & & & \\
\hline & & & & 125 & & & & & \\
\hline & & & & 126 & & & & & \\
\hline & Impulsive & 6 & 6 & 107 & 19.7 & 0.494 & 0.079 & 0.971 & 0.834 \\
\hline & & & & 109 & & & & & \\
\hline & & & & 112 & & & & & \\
\hline & & & & 114 & & & & & \\
\hline & & & & 115 & & & & & \\
\hline & & & & 116 & & & & & \\
\hline & Patient Role + Preoccupation & 11 & 10 & 117 & 39.6 & 0.233 & 0.070 & 0.960 & 0.864 \\
\hline & with health & & & 118 & & & & & \\
\hline & & & & 120 & & & & & \\
\hline & & & & 121 & & & & & \\
\hline & & & & 122 & & & & & \\
\hline & & & & 123 & & & & & \\
\hline & & & & 124 & & & & & \\
\hline & & & & 127 & & & & & \\
\hline & & & & 128 & & & & & \\
\hline & & & & 129 & & & & & \\
\hline & Personal Relations & 3 & 3 & 99 & 0.7 & 0.984 & & & 0.757 \\
\hline & & & & 100 & & & & & \\
\hline & & & & 101 & & & & & \\
\hline
\end{tabular}


All five accepted subscales were tested for internal consistency with Cronbach's alpha coefficients described in Table 5. None had an alpha below 0.7.

\section{Discussion}

\section{Major findings}

A measurement tool, which covers psychosocial experiences after the diagnosis of hypertension, was developed and validated, encompassing a total of 82 items divided into two parts and 15 subscales (10 in Part 1 and five in Part 2). We established known-group validity for the total score and proved that the instrument discriminates well between cases and controls.

The final scale is a multidimensional group of subscales, which, in turn, are unidimensional. By dividing the multidimensional scale in unidimensional subscales, we identified the key elements of the psychosocial consequences (a multidimensional construct by definition) to provide content coverage and relevance. We also measured each element within their own unidimensional subscale.

This study revealed that being labelled with hypertension has common psychosocial consequences with having abnormal screening results for breast cancer, lung cancer, cervical cancer and aortic aneurism, all of which were previous targets of the four different COS versions $[25,27,28,42]$. This finding is supported by the inclusion of COS 'disease-specific' items, which were accepted in the final version of the questionnaire. These results may also provide a comparison between the psychosocial consequences of labelling hypertension and the psychosocial consequences related to false positive results of screening related to such four conditions.

However, we do not expect that the new questionnaire, which is composed of new and inherited items from the COS family, is the same metric as the COS questionnaires. New items were generated, and they expanded the final version of the questionnaire, altering the composition of the item sets inherited from COS and thus measuring a different (but with similarities) construct from the COS versions. Hence, the psychosocial effects of labelling hypertension share similarities with the effects of being screened but are, to an extent, different from the other psychosocial effects measured by the COS questionnaires.

New subscales specifically relevant for people labelled with hypertension were developed. The subscale 'relations negative' strengthens the social aspects of the psychosocial consequences of labelling, whereas the subscale 'patient role' strengthens the labelling effects, suggesting that the labelled people develop actions and attitudes expected from the labelled condition. These relevant aspects are found in the qualitative content analysis of our previous study [12].
The scores generated from questionnaire scales are further valid if analyses based on item response theory (IRT) are conducted [30, 43, 44]. We used Rasch model analysis, one subgroup of IRT models. The selection of Rasch model analysis allowed us to start from our qualitatively developed domains, submit them in a survey and test if the response data fit the Rasch model [30].

All items were excluded using a data driven method. However, we found a strength, that is, our statistical psychometric analyses were not purely exploratory, but mostly confirmatory. We used Rasch model analysis to confirm our hypotheses: items were relevant, covered different aspects of the target outcome and worked well together. We referred to the qualitative material to analyse the impact of the exclusion on the subscales' content coverage and to explore possibilities to fix the excluded items' issues. Given that the developed subscales had adequate psychometric properties and enough items to allow for adequate content coverage, the excluded items may have their revised versions retested in the future.

The exclusion of items based on LD and DIF aim at including only items that are correlated through the latent trait, in this case, the psychosocial consequences of labelling hypertension composed of its identified subdimensions.

Traditionally, questionnaires are validated using analyses that are based on classical test theories, such as Cronbach's alpha and CFA. These methods are insufficient to establish unidimensionality [45], but can be used complementarily to support the Rasch model analysis results. In this study, the derived subscales were confirmed using CFA but should ideally be confirmed in a new dataset. The overall result is that the CFA models confirmed the measurement models derived using Rasch model analysis. Internal consistency reliability was also confirmed for most of the subscales. However, two of them, 'lifestyle' and 'sense of dejection', had values of Cronbach's coefficient alpha below 0.7, suggesting that they lack reliability. These subscales should be reviewed in the future. We also tested reliability with the sum of the scores of each sub-dimension for each of the questionnaire's part with Cronbach's alpha above 0.85 , indicating that the subscales work well together.

The final set of items was composed of a long questionnaire, which might not be of practical use. If it proves to be a problem for future use, the 11 single items can be excluded because they also make the results difficult to interpret. Moreover, subscales that are composed of more than five items can be easily shortened to produce an easy-to-apply questionnaire. Long questionnaires may provide improved content validity and identify nuance in the psychosocial consequences of labelling hypertension. 
Future studies can aim to disclose floor/ceiling effects, supporting the qualitative evaluation of content coverage.

This study has certain limitations. Considering that the questionnaire was distributed online (mobile and personal computer), making clarifications whilst completing the items was difficult (although available) for the participants. In a scenario with a wide range of reading abilities, a self-applied questionnaire can be less accessible. Certain items also showed DIF with gender and age, indicating that when using this scale, we must be careful when comparing the effects between male and female and people with different ages. Item 5 was found to be wrongly translated during the analysis. Therefore, further tests are recommended for this item in the 'emotional' dimension. Another recommendation is to retest the 'behaviour' dimension without this item. Note that Item 14 in Part 2 was never tested.

Another limitation of this study is that the sampling was based on an open design because no control existed on whether the subjects had really undergone a diagnosis of hypertension; specifically, a diagnosis of mild hypertension. We intended to measure the impact of labelling and assumed that such an effect requires the subject to recognise himself or herself as hypertensive, and not that the correct diagnosis is clinically identified. This assumption is justifiable because previous literature and our own qualitative findings in previous steps of the development of this questionnaire suggested that the effect of hypertension labelling is unrelated to the correct diagnosis of hypertension [4]. Furthermore, the prevalence of mild hypertension among people without comorbidities is far greater than that of moderate and severe hypertension [46]. When we included only those without comorbidities, we expected to remove most people with moderate and severe hypertension.

\section{Conclusion}

A new condition-specific questionnaire with a total of 82 items in 15 subscales was developed for people labelled with hypertension; the questionnaire had high content validity and adequate psychometric properties. This measure is called 'Consequences of Labelling Hypertension Questionnaire', which covers two parts of the psychosocial experiences after the diagnosis of hypertension. The adequate reliability, unidimensionality and invariant measurement of the subscales were demonstrated using Rasch model analysis. However, further examinations are required for the final subscales in a new dataset to confirm the results presented here and promote improvements to this questionnaire.

\section{Implications for clinical practice and research}

This questionnaire is not designed to be used in clinical practice. However, research on the psychosocial consequences of labelling is relevant for clinical practice and for population studies. It is a tool that can be used in future research on hypertension, especially in scenarios of screening, preventive population strategies and in intervention studies that are willing to access all possible results of the interventions.

\section{Supplementary Information}

The online version contains supplementary material available at https://doi. org/10.1186/s41687-021-00291-4.

Additional file 1. Plots: observed mean score in each class interval (line) and the $95 \%$ confidence region of the model expectations (shaded area).

\section{Abbreviations}

CVD: Cardiovascular disease; COS: Consequences of screening; PROM: Patient reported outcome measure; IRT: Item response theory; CFA: Confirmatory factor analysis; LD: Local dependence; DIF: Differential item functioning

\section{Acknowledgements}

We thank all the participants in the research. We specially thank Lucas Bastos and Volkert Siersma for great contributions. JG specially thanks all the opportunities given by his supervisors.

\section{Authors' contributions}

$J G, J B$ and $A O$ designed the study. JG conducted the survey. KC, JB and JG conducted the psychometric analysis. JG drafted the manuscript. KC, AO and JB contributed to revisions with important intellectual content. All authors accepted the final version of the manuscript. All authors had full access to all data in the study and take responsibility for the integrity of the data and the accuracy of the data analysis. JG is guarantor. The authors read and approved the final manuscript.

\section{Authors' information}

Three of the authors are clinicians in General Practice and one is a biostatistician. JG had no previous experience with psychometric analysis. KC and JB are experienced researchers in psychometric analysis methods. An English ad hoc translation of the empirical material was shared with JB and KC, who do not read or speak Brazilian Portuguese. JB and KC are Danish and $J G$, and $A O$ are Brazilians.

\section{Funding}

The first author was granted a 1 year scholarship by CAPES (Brazil) for completing a 1 year visit to Københavns Universitet during his Ph.D. programme in Brazil. Award number: 88881.132103/2016-01.

\section{Availability of data and materials}

The datasets used and/or analyzed during the current study are available from the corresponding author on reasonable request.

\section{Ethics approval and consent to participate}

All informants provided their informed consent, and the study was approved by the Faculdade de Medicina da Universidade de São Paulo ethical committee, CAAE 54699716.0.0000.0065.

\section{Consent for publication}

Not applicable.

\section{Competing interests}

The authors declare that they have no competing interests.

\section{Author details}

${ }^{1}$ Departamento de Medicina Preventiva, Faculdade de Medicina da Universidade de São Paulo, Av. Dr Arnaldo, 4552 andar. CEP, São Paulo, SP 01246-903, Brazil. '2Department of Public Health, Section of General Practice and Research Unit for General Practice, University of Copenhagen, Øster Farimagsgade 5, building 24, P.O. Box 2099, 1014 Copenhagen K, Denmark. ${ }^{3}$ Department of Public Health, Section of Biostatistics, University of 
Copenhagen, Øster Farimagsgade 5, Building 15, P.O. Box 2099, 1014 København K, Denmark. ${ }^{4}$ Primary Health Care Research Unit, Copenhagen, Region Zealand, Denmark.

Received: 28 September 2019 Accepted: 19 January 2021

Published online: 04 February 2021

\section{References}

1. GBD 2015 Disease and Injury Incidence and Prevalence Collaborators . (2016). Global, regional, and national incidence, prevalence, and years lived with disability for 310 diseases and injuries, 1990-2015: A systematic analysis for the global burden of disease study 2015. Lancet, 388(10053), 1545-1602. https://doi.org/10.1016/s0140-6736(16)31678-6.

2. Vinther, J. L., Jorgensen, T., \& Borglykke, A. (2013). Need to combine individual strategies with population-level strategies in the prevention of coronary heart disease. Danish Medical Journal, 60(10), A4726.

3. Krogsboll, L. T., Jorgensen, K. J., \& Gotzsche, P. C. (2019). General health checks in adults for reducing morbidity and mortality from disease. Cochrane Database of Systematic Reviews, 1, CD009009.

4. Ogedegbe, G. (2010). Labeling and hypertension: It is time to intervene on its negative consequences. Hypertension., 56(3), 344-345.

5. Pickering, G. (1978). Hypertension in general practice. Journal of the Royal Society of Medicine, $71(12), 885-889$.

6. Macdonald, L. A., Sackett, D. L., Haynes, R. B., \& Taylor, D. W. (1984). Labelling in hypertension: A review of the behavioural and psychological consequences. Journal of Chronic Diseases, 37(12), 933-942.

7. Haynes, R. B., Sackett, D. L., Taylor, D. W., Gibson, E. S., \& Johnson, A. L. (1978), Increased absenteeism from work after detection and labeling of hypertensive patients. The New England Journal of Medicine, 299(14), 741-744.

8. Mossey, J. M. (1981). Psychosocial consequences of labelling in hypertension. Clinical and Investigative Medicine, 4(3-4), 201-207.

9. Snyder, C. F., Jensen, R. E., Segal, J. B., \& Wu, A. W. (2013). Patient-reported outcomes (PROs): Putting the patient perspective in patient-centered outcomes research. Medical Care, 51 (8 Suppl 3), S73-S79.

10. Brodersen, J., McKenna, S. P., Doward, L. C., \& Thorsen, H. (2007). Measuring the psychosocial consequences of screening. Health and Quality of Life Outcomes, 5, 3.

11. Sangren, H., Reventlow, S., \& Hetlevik, I. (2009). Role of biographical experience and bodily sensations in patients' adaptation to hypertension. Patient Education and Counseling, 74(2), 236-243.

12. Gyuricza, J. V., d'Oliveira, A., Machado, L. B. M., \& Brodersen, J. (2019). Development of an item pool for a questionnaire on the psychosocia consequences of hypertension labelling. Journal of Patient-Reported Outcomes, 4(1), 2.

13. Hamer, M., Batty, G. D., Stamatakis, E., \& Kivimaki, M. (2010). Hypertension awareness and psychological distress. Hypertension., 56(3), 547-550.

14. Goldberg, D. P., \& Hillier, V. F. (1979). A scaled version of the general health questionnaire. Psychological Medicine, 9(1), 139-145.

15. Côté, I., Grégoire, J.-P., Moisan, J., \& Chabot, I. (2004). Quality of life in hypertension: The SF-12 compared to the SF-36. The Canadian journal of clinical pharmacology = Journal canadien de pharmacologie clinique, 11, e232-e238.

16. Wiebe, S., Guyatt, G., Weaver, B., Matijevic, S., \& Sidwell, C. (2003). Comparative responsiveness of generic and specific quality-of-life instruments. Journal of Clinical Epidemiology, 56(1), 52-60.

17. Hobart, J. C., Williams, L. S., Moran, K., \& Thompson, A. J. (2002). Quality of life measurement after stroke: Uses and abuses of the SF-36. Stroke., 33(5), 1348-1356.

18. Comins, J., Brodersen, J., Krogsgaard, M., \& Beyer, N. (2008). Rasch analysis of the knee injury and osteoarthritis outcome score (KOOS): A statistical reevaluation. Scandinavian Journal of Medicine \& Science in Sports, 18(3), 336-345.

19. Hagell, P., Reimer, J., \& Nyberg, P. (2009). Whose quality of life? Ethical implications in patient-reported health outcome measurement. Value in Health, 12(4), 613-617.

20. Tennant, A., \& McKenna, S. P. (1995). Conceptualizing and defining outcome. British Journal of Rheumatology, 34(10), 899-900.

21. Nguyen, T. H., Han, H. R., Kim, M. T., \& Chan, K. S. (2014). An introduction to item response theory for patient-reported outcome measurement. Patient., 7(1), 23-35.

22. Brodersen, J., Doward, L. C., Thorsen, H., \& McKenna, S. P. (2013). Writing health-related items for Rasch models - patient-reported outcome scales for health sciences: From medical paternalism to patient autonomy. In K. B. Christensen, S. Kreiner, \& M. Mesbah (Eds.), Rasch models in health. Applied mathematics series, (pp. 281-298). London: ISTE Ltd..

23. Brodersen, J., Thorsen, H., \& Cockburn, J. (2004). The adequacy of measurement of short and long-term consequences of false-positive screening mammography. Journal of Medical Screening, 11(1), 39-44.

24. Andrich, D. (2004). Controversy and the Rasch model: A characteristic of incompatible paradigms? Medical Care, 42(1 Suppl), 17-116.

25. Brodersen, J., Siersma, V., \& Thorsen, H. (2018). Consequences of screening in cervical cancer: Development and dimensionality of a questionnaire. BMC Psychology, 6(1), 39

26. Brodersen, J., Hansson, A., Johansson, M., Siersma, V., Langenskiold, M., \& Pettersson, M. (2017). Consequences of screening in abdominal aortic aneurysm: Development and dimensionality of a questionnaire. Journal of Patient-Reported Outcomes, 2, 37.

27. Brodersen, J., Thorsen, H., \& Kreiner, S. (2010). Consequences of screening in lung cancer: Development and dimensionality of a questionnaire. Value in Health, 13(5), 601-612.

28. Brodersen, J., \& Thorsen, H. (2008). Consequences of screening in breast cancer (COS-BC): Development of a questionnaire. Scandinavian Journal of Primary Health Care, 26(4), 251-256.

29. Rasch, G. (1980). Probabilistic models for some intelligence and attainment tests. Chicago, University of Chicago Press.

30. Christensen, K. B., Kreiner, S., \& Mesbah, M. (2013). Rasch models in health, (p. xvi, 368). London, Hoboken: ISTE ; Wiley.

31. Christensen, K. B., Makransky, G., \& Horton, M. (2017). Critical values for Yen's Q3: Identification of local dependence in the Rasch model using residual correlations. Applied Psychological Measurement, 41(3), 178-194.

32. Holland, P. W., \& Wainer, H. (1993). Educational Testing Service. Differential item functioning, ( $p . x v, 453)$. Hillsdale: Lawrence Erlbaum Associates.

33. Brodersen, J., Meads, D., Kreiner, S., Thorsen, H., Doward, L., \& McKenna, S. (2007). Methodological aspects of differential item functioning in the Rasch model. Journal of Medical Economics, 10(3), 309-324.

34. Kreiner, S., \& Christensen, K. B. (2002). Graphical Rasch models. In M. Mesbah, B. F. Cole, \& M.-L. T. Lee (Eds.), Statistical methods for quality of life studies: Design, measurements and analysis, (pp. 187-203). Boston: Springer US.

35. Kreiner, S. (2007). Validity and objectivity: Reflections on the role and nature of Rasch models. Nordic Psychology, 59(3), 268-298.

36. Kreiner, S., \& Christensen, K. B. (2007). Validity and objectivity in health-related scales: Analysis by graphical Loglinear Rasch models. Multivariate and mixture distribution Rasch models: Extensions and applications, (pp. 329-346). New York: Springer New York.

37. Andersen, E. B. (1973). Conditional inference for multiple-choice questionnaires. The British Journal of Mathematical and Statistical Psychology, 26(1), 31-44.

38. Kreiner, S., \& Nielsen, T. (2013). Item analysis in DIGRAM: Guided tours. Research report 13/06. Copenhangen: Department of Biostatistics, University of Copenhagen.

39. Benjamini, Y., \& Hochberg, Y. (1995). Controlling the false discovery rate: A practical and powerful approach to multiple testing. Journal of the Royal Statistical Society: Series B: Methodological, 57(1), 289-300.

40. Hu, L.-t., \& Bentler, P. M. (1998). Fit indices in covariance structure modeling: Sensitivity to underparameterized model misspecification. Psychological Methods, 3(4), 424-453.

41. Cho, E., \& Kim, S. (2015). Cronbach's coefficient alpha:Well known but poorly understood. Organizational Research Methods, 18(2), 207-230.

42. Brodersen, J., Hansson, A., Johansson, M., Siersma, V., Langenskiöld, M., \& Pettersson, M. (2018). Consequences of screening in abdominal aortic aneurysm: Development and dimensionality of a questionnaire. Journal of Patient-Reported Outcomes, 2(1), 37.

43. Tennant, A., McKenna, S. P., \& Hagell, P. (2004). Application of Rasch analysis in the development and application of quality of life instruments. Value in Health, 7(Suppl 1), S22-S26.

44. Linden, W. J., \& Hambleton, R. K. (1997). Handbook of modern item response theory, (p. xv, 510). New York: Springer.

45. Wright, B. D., \& Mok, M. (2000). Rasch models overview. Journal of Applied Measurement, 1(1), 83-106.

46. Kaplan, R. M., \& Ong, M. (2007). Rationale and public health implications of changing CHD risk factor definitions. Annual Review of Public Health, 28, 321-344.

\section{Publisher's Note}

Springer Nature remains neutral with regard to jurisdictional claims in published maps and institutional affiliations. 